\title{
Cirugías por traumatismo torácico: Caracterización y variables asociadas a mortalidad
}

\author{
Roberto González L. ${ }^{1,2}$, Alejandra Riquelme U. ${ }^{3}$, Rodrigo Reyes M.., 2 , Felipe Alarcón O. ${ }^{4}$ \\ Sebastián Barra M. ${ }^{4}$, Enrique Seguel S. ${ }^{1,2}$, Aleck Stockins L. ${ }^{1,2}$, Andrés Jadue T. ${ }^{1}$, \\ Diego Saldivia Z. ${ }^{4}$, Andrés Schaub C. ${ }^{4}$ y Emilio Alarcón C. ${ }^{1,2}$
}

\section{Thoracic trauma surgery: indications, findings and mortality associated variables}

Background: Thoracic Trauma (TT) is the cause of approximately a quarter of trauma deaths. The patients who undergo Thoracic Trauma Surgery (TTS) present a wide spectrum of characteristics and prognosis. Aim: To describe clinical characteristics, indications, temporality, morbidity, mortality and mortality associated variables in TTS patients. Materials and Method: Observational study of TT hospitalized patients, period January-1981 to December-2019. A review of operation notes and database was done. A logistic regression for mortality associated variables was made. To compare classification, type of TT and its temporal distribution, SPSS $25^{\circledR}$ with chi-square test was used, considering significant $\mathrm{p}<0.05$. Results: A total of $808(18.2 \%)$ of 4.448 TT patients required TTS, 767 (94.9\%) were men with average age: $31.5 \pm$ 13.8. The trauma was penetrating trauma due to a stab in most cases, $164(20.3 \%)$ were polytraumatized. The surgery was urgent in 474 (58.7\%), early in $41(5.0 \%)$ and delayed in $293(36.3 \%)$ cases. The global mortality was $6.7 \%$ and was significantly higher in the blunt TT, polytrauma, urgent and early surgery patients. Mortality in urgent TTS was $9.7 \%$, early $4.9 \%$ and $2.0 \%$ in delayed $(p<0.001)$. Independent variables associated with mortality were observed. Conclusions: In our series, TTS were performed mainly in young men with penetrating TT. The group was heterogeneous regarding surgical indications, findings and intrathoracic or associated injuries. Multiple variables showed to influence significantly on mortality in patients who underwent TTS.

Keywords: Thoracic injuries; thoracic surgery; wounds and injuries; thoracotomy; mortality determinants.

\section{Resumen}

Introducción: El traumatismo torácico (TT) es la causa de aproximadamente un cuarto de las muertes por traumatismos. Los pacientes tratados con cirugía por traumatismo torácico (CTT) presentan un amplio espectro de características y pronósticos. Objetivos: Describir características clínicas, indicaciones, temporalidad, morbilidad, mortalidad y las variables asociadas a mortalidad en pacientes con CTT. Materiales y Método: Estudio observacional de pacientes tratados con CTT, período enero-1981 a diciembre-2019. Revisión de protocolos prospectivos de TT y base de datos. Se realizó regresión logística para variables asociadas a mortalidad. Se utilizó SPSS $25^{\circledR}$ con prueba chi-cuadrado para comparar clasificación, tipo de TT y su distribución temporal, considerando significativo $p<0,05$. Resultados: En total 808 casos $(18,2 \%)$ de 4.448 TT requirieron CTT. Fueron hombres 767 (94,9\%) y la edad promedio fue 31,5 $\pm 13,8$ años. El traumatismo fue penetrante y por arma blanca en la mayoría de los casos. Fueron politraumatizados 164 (20,3\%). La cirugía fue urgente en 474 (58,7\%), precoz en 41 (5,0\%) y diferida en $293(36,3 \%)$ casos. La mortalidad global fue de $6,7 \%$ y fue significativamente mayor en TT contusos, politraumatizados y en cirugía urgente. La mortalidad fue $9,7 \%$ en CTT urgente, 4,9\% en precoz y 2,0\% en diferida ( $p<0,001)$. Se observaron variables independientes asociadas a mortalidad. Conclusión: En nuestra serie, las CTT se realizaron principalmente en hombres jóvenes con TT penetrantes. Correspondieron a un grupo heterogéneo en cuanto a las indicaciones, hallazgos y lesiones intratorácicas y/o asociadas. Múltiples variables demostraron influir significativamente en la mortalidad de los pacientes tratados con CTT.

Palabras clave: traumatismos torácicos; cirugía torácica; heridas y traumatismos; toracotomía; factores determinantes de mortalidad.
Centro Cardiovascular, Hospital Clínico Regional de Concepción Dr. Guillermo Grant Benavente. Concepción Chile.

2Departamento de Cirugía, Facultad de Medicina,

Universidad de Concepción. Concepción, Chile. 3Servicio de Cirugía, Hospital Clínico Regional de Concepción Dr. Guillermo Grant Benavente. Concepción, Chile.

${ }^{4}$ Estudiante de Medicina, Facultad de Medicina, Universidad de Concepción. Concepción, Chile.

Recibido 2020-10-14 aceptado 2020-12-09

Correspondencia a: Dr. Roberto González L. rgonzalezlagos@udec.cl 


\section{Introducción}

El traumatismo es la causa de alrededor de cinco millones de muertes cada año, atribuyéndose al traumatismo torácico (TT) un 20\%-25\% de estas. El TT ocupa el tercer lugar en frecuencia y constituye aproximadamente un $10 \%$ de las hospitalizaciones por traumatismos ${ }^{1-3}$.

El TT requiere una cirugía mayor en aproximadamente un 5\%-15\% de los casos ${ }^{4-7}$. De este modo, los tratamientos más frecuentes son la pleurotomía y el tratamiento médico exclusivo. Los distintos mecanismos del traumatismo y el amplio espectro de las lesiones derivadas del TT otorgan a la cirugía por traumatismo torácico (CTT) una importante heterogeneidad, incluyendo desde intervenciones quirúrgicas inmediatas en pacientes con paro cardiorrespiratorio, como la toracotomía de emergencia, hasta cirugías diferidas por complicaciones pleurales postraumáticas tardías.

Análisis de pacientes tratados con CTT han demostrado variaciones en las indicaciones, abordajes y la temporalidad de la cirugía dependiendo de las características de los traumatismos ${ }^{5,8}$. Estas influyen en el pronóstico, siendo relevantes al momento de evaluar los resultados de las cirugías en estos pacientes. En nuestro medio existe escasa información sobre la CTT.

Nuestros objetivos son describir las características clínicas, indicaciones, temporalidad, morbilidad, mortalidad y las variables asociadas a mortalidad en pacientes con CTT.

\section{Materiales y Método}

Se realizó un estudio observacional que incluyó a todos los pacientes con CTT tratados por el equipo de Cirugía Cardiotorácica del Hospital Clínico Regional "Dr. Guillermo Grant Benavente" de Concepción, Chile, en el período enero-1981 a diciembre-2019. La información se obtuvo a partir de protocolos prospectivos de TT realizados al momento del ingreso, revisión de base de datos del Equipo de Cirugía Cardiotorácica, protocolos quirúrgicos y fichas clínicas.

Se calcularon los índices de gravedad del traumatismo (IGT): ISS ${ }^{9}$ (Injury Severity Score), RTS-T ${ }^{10}$ (Revised Trauma Score Triage) y TRISS ${ }^{11}$ (Trauma Injurity Severity Score), que corresponden a índices basados en lesión anatómica, parámetros fisiológicos y mixto (anatómico-fisiológico), respectivamente. Se consideraron politraumatizados los pacientes con un puntaje ISS $>16$ puntos $^{12}$.
La temporalidad de la cirugía se clasificó según el tiempo transcurrido entre el ingreso del paciente al servicio de urgencias y el inicio de la CTT considerándose urgente $\leq 4 \mathrm{~h}$, precoz $>4-24 \mathrm{~h}$ y diferida $>24 \mathrm{~h}^{13}$.

Para simplificar el análisis, las causas de muerte fueron descritas de forma sindromática y se agruparon las muertes producidas por el síndrome de respuesta inflamatoria sistémica (SIRS) y la falla orgánica múltiple (FOM) en una misma categoría ${ }^{14,15}$.

Se describe la edad, sexo y características clínicas de los pacientes, mecanismos, tipo, IGT y tratamiento del TT, indicaciones quirúrgicas y temporalidad de la cirugía, hallazgos y/o lesiones, morbilidad y mortalidad.

Se comparó la mortalidad observada según la temporalidad de la cirugía, la presencia de lesiones asociadas y el tipo de TT mediante la prueba de chicuadrado y exacta de Fisher según corresponda. Se realizó un análisis univariado de las variables asociadas a mortalidad y, posteriormente, se realizó una regresión logística multivariada con las variables que mostraron asociación significativa en el primer análisis. Se calculó Odds Ratio (OR) y un intervalo de confianza del 95\%. Para el análisis estadístico se utilizó el programa estadístico $S P S S^{\circledR}$ v25 y se consideró significativo un valor $\mathrm{p}<0,05$.

El estudio y análisis de las hospitalizaciones por TT fue aprobado por el comité ético-científico de nuestra institución.

\section{Resultados}

Durante el periodo fueron hospitalizados 4.448 pacientes por TT de los cuales en $808(18,2 \%)$ se realizó una CTT.

En los pacientes con CTT, la edad promedio fue de 31,5 $\pm 13,8$ años, predominó en hombres, en 767 $(94,9 \%)$ con una relación hombre/mujer de 18,7/1. El principal agente del traumatismo fue el arma blanca, en $558(69,1 \%)$ casos y los mecanismos traumáticos más frecuentes fueron la agresión y los accidentes de tránsito, en $620(76,7 \%)$ y $92(11,4 \%)$ casos respectivamente. El tipo de traumatismo fue penetrante en $652(80,7 \%)$ casos. En cuanto a las lesiones asociadas, se consideró TT aislado en $600(74,3 \%)$, con lesiones asociadas $208(25,7 \%)$, y de estos, politraumatismo en $164(20,3 \%)$ casos (Tabla 1).

Las indicaciones quirúrgicas más frecuentes fueron el traumatismo penetrante cardíaco (TPC) en $235(24,8 \%)$ y las complicaciones del hemotórax (coagulado, retenido, tabicado y/o persistente) en $201(21,2 \%)$ pacientes (Tabla 2$)$. 
Tabla 1. Edad, sexo, agente, clasificación, mecanismo y tipo de traumatismo en pacientes con cirugías por traumatismo torácico

\begin{tabular}{|lc|}
\hline Total cirugías por TT & $\mathbf{8 0 8}$ \\
Edad & \\
Edad promedio en años & $31,5 \pm 13,8$ \\
Mediana & 28 \\
Sexo & \\
Hombre & $767(94,9 \%)$ \\
Mujer & $41 \quad(5,1 \%)$ \\
Agente del trauma & \\
Arma blanca & $558(69,1 \%)$ \\
Vehículo motorizado & $94(11,6 \%)$ \\
Arma de fuego & $71(8,8 \%)$ \\
Elemento contuso & $52(6,4 \%)$ \\
Otros agentes & $33(4,1 \%)$ \\
Clasificación & \\
Penetrante & $652(80,7 \%)$ \\
Contuso & $156(19,3 \%)$ \\
Mecanismo & \\
Agresión & $620(76,7 \%)$ \\
Accidente de tránsito & $92(11,4 \%)$ \\
Iatrogenia & $22(2,7 \%)$ \\
Accidente doméstico & $19(2,4 \%)$ \\
Accidente laboral & $19(2,4 \%)$ \\
Autoagresión & $12(1,5 \%)$ \\
Otro mecanismo & $24(3,0 \%)$ \\
Tipo de traumatismo & $600(74,3 \%)$ \\
TT aislado & $208(25,7 \%)$ \\
TT con lesiones asociadas & $164(20,3 \%)$ \\
$\quad$ Politraumatismo & \\
\hline Traumatismo toracico & \\
\hline
\end{tabular}

TT: Traumatismo torácico.

En relación con la temporalidad de las CTT, la mayoría fueron urgentes, en $474(58,7 \%)$ casos y se realizó cirugía extratorácica en $119(14,7 \%)$ casos. La necesidad posoperatoria de ventilación mecánica y de transfusiones se detallan en la Tabla 3.

Los índices de gravedad fueron en promedio: ISS $17,9 \pm 11,0$ puntos, RTS-T $10,6 \pm 2,5$ puntos y TRISS (mortalidad esperada) de 11,5. La cirugía urgente y el politraumatismo fueron subgrupos que demostraron una mayor gravedad según los IGT y una mayor mortalidad observada (Tabla 4).

La mortalidad global de las CTT fue del 6,7\%, que ocurrió en su mayoría dentro de los primeros 7 días desde el ingreso, en 43 (79,6\%) casos. Durante los primeros 7 días de hospitalización la causa de muerte más frecuente fue el shock hipovolémico
Tabla 2. Indicaciones quirúrgicas de pacientes con cirugía por traumatismo torácico

\begin{tabular}{|lr|}
\hline Total cirugías por traumatismo torácico & $\mathbf{8 0 8}$ \\
Indicaciones principales* & \\
Traumatismo penetrante cardíaco & $235(24,8 \%)$ \\
Complicación del hemotórax** & $201(21,2 \%)$ \\
Hemotórax masivo & $125(13,2 \%)$ \\
Lesión diafragmática & $86(9,1 \%)$ \\
Lesión de parénquima pulmonar & $75(7,9 \%)$ \\
Lesión de grandes vasos & $71(7,5 \%)$ \\
Lesión y/o estabilización de pared torácica & $69(7,3 \%)$ \\
Empiema y/o hemotórax infectado & $58(7,2 \%)$ \\
Lesión de vía aérea & $7(0,7 \%)$ \\
Otras & $22(2,3 \%)$ \\
Cirugía mínimamente invasiva*** & $77(9,5 \%)$ \\
Complicación del hemotórax** & $65(84,4 \%)$ \\
Empiema y/o hemotórax infectado & $8(10,4 \%)$ \\
Lesión de arteria mamaria interna & $2(2,6 \%)$ \\
Lesión diafragmática & $1(1,3 \%)$ \\
Lesión de parénquima pulmonar & $1(1,3 \%)$ \\
\hline
\end{tabular}

TT: Traumatismo torácico. *Se registraron casos con dos o más indicaciones. **Hemotórax coagulado, retenido, tabicado y/o persistente. $* * *$ Cirugía torácica videoasistida (VATS) o videotoracoscopía (VTC). Todas las cirugías mínimamente invasivas fueron diferidas $(>24 \mathrm{~h})$.

Tabla 3. Temporalidad de la cirugía, pleurotomía preoperatoria, ventilación mecánica posoperatoria, cirugía extratorácica y transfusiones en pacientes con cirugía por traumatismo torácico

\begin{tabular}{|lc|}
\hline Total cirugías por traumatismo torácico & $\mathbf{8 0 8}$ \\
Temporalidad de la cirugía & \\
Urgente $(\leq 4 \mathrm{~h})$ & $474(58,7 \%)$ \\
Precoz $(>4-24 \mathrm{~h})$ & $41(5,0 \%)$ \\
Diferida $(>24 \mathrm{~h})$ & $293(36,3 \%)$ \\
Pleurotomía preoperatoria* & $638(78,9 \%)$ \\
Ventilación mecánica posoperatoria & $118(14,6 \%)$ \\
Cirugía extratorácica asociada** & $119(14,7 \%)$ \\
Abdominal & $104(12,9 \%)$ \\
Vascular & $16(2,0 \%)$ \\
Traumatológica & $10(1,2 \%)$ \\
Otras & $7(0,9 \%)$ \\
Transfusiones & $211(26,1 \%)$ \\
Hemoderivados & \\
$\quad$ Promedio (unidades por paciente transfundido) & $8,3 \pm 8,9$ \\
Mediana & 4 \\
Glóbulos rojos & \\
$\quad$ Promedio (unidades por paciente transfundido) & $5,2 \pm 4,1$ \\
$\quad$ Mediana & 4 \\
\hline
\end{tabular}

*En la serie global de 4.448 pacientes hospitalizados con traumatismo torácico, 2.510 casos fueron tratados con pleurotomía por hemotórax, neumotórax y/o hemoneumotórax, sin otra lesión con indicación quirúrgica torácica per sé. De estos, en $150(6 \%)$ se realizó cirugía torácica por disfunción, complicación o porque la pleurotomía fue insuficiente como tratamiento. En este grupo de pacientes tratados con pleurotomía la mediana de estadía hospitalaria fue de 4 días. ${ }^{* * S e}$ registraron casos con dos o más cirugías extratorácicas. 
atribuyéndose a $32(74,4 \%)$ casos, mientras que la mortalidad posterior a la primera semana fue en $\mathrm{su}$ mayoría por SIRS/FOM o sepsis (Tabla 5).

Los hallazgos y/o lesiones intratorácicas más frecuentes fueron el hemotórax no masivo $(59,5 \%)$, el neumotórax $(33,3 \%)$ y el TPC $(29,1 \%)$. En cuanto a las lesiones extratorácicas, el traumatismo concomitante más frecuente fue el traumatismo abdominal en $123(15,2 \%)$ casos (Tabla 6).

Tabla 4. Índices de gravedad del traumatismo según temporalidad y tipo de traumatismo en pacientes con cirugía por traumatismo torácico

\begin{tabular}{|lcccc|}
\hline & ISS & RTS-T & TRISS & Mortalidad \\
Total cirugías por TT & $17,9 \pm 11,0$ & $10,6 \pm 2,5$ & 11,5 & $6,7 \%$ \\
Temporalidad & & & & \\
Urgente ( $\leq 4 \mathrm{~h})$ & $20,7 \pm 11,7$ & $10,1 \pm 2,9$ & 15,8 & $9,7 \%$ \\
Precoz (> 4-24 h) & $18,3 \pm 14,4$ & $11,0 \pm 2,3$ & 10,0 & $4,9 \%$ \\
Diferido (> 24 h) & $13,3 \pm 7,3$ & $11,3 \pm 1,7$ & 4,6 & $2,0 \%$ \\
Tipo de traumatismo & & & & \\
TT aislado & $16,0 \pm 10,7$ & $10,7 \pm 2,4$ & 10,2 & $5,0 \%$ \\
TT con lesiones asociadas & $23,4 \pm 10,2$ & $10,3 \pm 2,7$ & 15,3 & $11,5 \%$ \\
$\quad$ Politraumatismo & $26,5 \pm 9,1$ & $10,0 \pm 3,0$ & 18,6 & $13,4 \%$ \\
\hline
\end{tabular}

TT: Traumatismo torácico; ISS: Injury Severity Score; RTS-T: Revised Trauma Score-Triage; TRISS: Trauma Injury Severity Score.

Tabla 5. Causa principal a la cual se atribuyó la mortalidad y temporalidad de la mortalidad en pacientes con cirugías por traumatismo torácico

\begin{tabular}{|lr|}
\hline Total cirugías por traumatismo torácico & $\mathbf{8 0 8}$ \\
Causa principal de mortalidad & $54(6,7 \%)$ \\
Shock hipovolémico & $32(59,3 \%)$ \\
SIRS/FOM & $11(20,4 \%)$ \\
Sepsis & $6(11,1 \%)$ \\
Otros & $5(9,2 \%)$ \\
Mortalidad a 0 - 7 días & $43(79,6 \%)$ \\
Shock hipovolémico & $32(74,4 \%)$ \\
SIRS/FOM & $6(14,0 \%)$ \\
Sepsis & $2(4,6 \%)$ \\
Otros & $3(7,0 \%)$ \\
Mortalidad a 8 - 30 días & $9(16,7 \%)$ \\
SIRS/FOM & $4(44,4 \%)$ \\
Sepsis & $3(33,3 \%)$ \\
Otros & $2(22,3 \%)$ \\
Mortalidad $>30$ días & $2(3,7 \%)$ \\
Sepsis & $1(50,0 \%)$ \\
SIRS/FOM & $1(50,0 \%)$ \\
\hline
\end{tabular}

SIRS: Síndrome de respuesta inflamatoria sistémica; FOM: Falla orgánica múltiple.
En relación con la CTT, $84(10,4 \%)$ pacientes presentaron morbilidad atribuida a la cirugía, 48 $(5,9 \%)$ casos requirieron reoperación torácica y 16 $(2,0 \%)$ reoperación extratorácica. La estadía posoperatoria promedio fue de $9,8 \pm 10,5$ días, con una mediana de 7 días (Tabla 7).

Al comparar la mortalidad en subgrupos según la temporalidad de la CTT, lesiones asociadas y el tipo de TT, se observaron diferencias significativas,

Tabla 6. Hallazgos y/o lesiones intratorácicas principales y extratorácicas en pacientes con cirugía por traumatismo torácico

\begin{tabular}{|c|c|}
\hline $\begin{array}{l}\text { Total cirugías por traumatismo } \\
\text { torácico }\end{array}$ & 808 \\
\hline \multicolumn{2}{|l|}{$\begin{array}{l}\text { Hallazgos y/o lesiones intratorácicas } \\
\text { principales* }\end{array}$} \\
\hline Hemotórax no masivo & $481(59,5 \%)$ \\
\hline Neumotórax & $269(33,3 \%)$ \\
\hline Penetrante cardiaca & $235(29,1 \%)$ \\
\hline Fracturas costales $* *$ & $150(18,6 \%)$ \\
\hline Hemotórax masivo & $125(15,5 \%)$ \\
\hline Enfisema subcutáneo & $122(15,1 \%)$ \\
\hline Lesión de parénquima pulmonar & $101(12,5 \%)$ \\
\hline Lesión diafragmática & $91(11,3 \%)$ \\
\hline Contusión pulmonar & $88(10,9 \%)$ \\
\hline Lesión de grandes vasos & $78 \quad(9,7 \%)$ \\
\hline Empiema y/o hemotórax infectado & $58 \quad(7,2 \%)$ \\
\hline Lesión de arteria mamaria & $29(3,6 \%)$ \\
\hline Lesión de pared torácica & $22(2,7 \%)$ \\
\hline Neumotórax hipertensivo & $15(1,9 \%)$ \\
\hline Traumatopnea & $15(1,9 \%)$ \\
\hline Fractura esternal & $13(1,6 \%)$ \\
\hline Lesión de vía aérea & $7(0,9 \%)$ \\
\hline Lesión de plexo braquial & $6(0,7 \%)$ \\
\hline Rotura esofágica & $4(0,5 \%)$ \\
\hline Luxación esternal & $1 \quad(0,1 \%)$ \\
\hline \multicolumn{2}{|l|}{ Lesiones extratorácicas asociadas* } \\
\hline Abdominales & $123(15,2 \%)$ \\
\hline Craneoencefálicas & $51 \quad(6,3 \%)$ \\
\hline Extremidades & $49(6,1 \%)$ \\
\hline Partes blandas & $20(2,5 \%)$ \\
\hline Fractura de pelvis & $15(1,9 \%)$ \\
\hline Faciales & $5(0,6 \%)$ \\
\hline
\end{tabular}

*Se registraron casos con dos o más hallazgos y/o lesiones intratorácicas y/o extratorácicos. **Se incluyó tórax volante. 
presentando la mayor mortalidad el subgrupo de pacientes con TT contuso, politraumatizado y cuya cirugía fue urgente, observándose una mortalidad del $26,2 \%$ en este grupo (Tablas 8 y 9; Figuras $1 \mathrm{~A}$ y 1B).

En el análisis multivariado fueron variables independientemente asociadas a mortalidad el TT contuso, el TPC, el deterioro fisiológico y el shock al ingreso, el TT por arma de fuego, el politraumatismo grave, entre otros (Figuras 2A y 2B).

\section{Discusión}

En nuestra serie, los pacientes tratados con CTT correspondieron principalmente a hombres jóvenes con TT penetrante. La CTT fue urgente en la mayoría de los casos y fue secundaria principalmente al TPC y el hemotórax masivo, mientras que las cirugías diferidas se realizaron más frecuentemente por complicaciones tardías del hemotórax. La mortalidad de los pacientes con CTT varió significativamente según el tipo de traumatismo, la temporalidad de la cirugía y las lesiones asociadas. Entre otras variables asociadas a mortalidad, destacamos el deterioro del estado fisiológico al ingreso y el politraumatismo grave.
Tabla 7. Morbilidad, reoperaciones, días de hospitalización y reingreso en pacientes con cirugía por traumatismo torácico

\begin{tabular}{|lc|}
\hline Total cirugías por traumatismo torácico & $\mathbf{8 0 8}$ \\
Morbilidad de cirugía torácica* & $84(10,4 \%)$ \\
Hemotórax complicado & $25(3,1 \%)$ \\
Infección herida quirúrgica & $22(2,7 \%)$ \\
Hemorragia posoperatoria & $16(2,0 \%)$ \\
Empiema & $10(1,2 \%)$ \\
Otros & $26(3,2 \%)$ \\
Reoperación & \\
Torácica & $48(5,9 \%)$ \\
Extratorácica & $16(2,0 \%)$ \\
Estadía hospitalaria (días) & \\
Promedio & $12,0 \pm 11,3$ \\
Mediana & 9 \\
Rango & $1-150$ \\
Estadía posoperatoria (días) & \\
Promedio & $9,8 \pm 10,5$ \\
Mediana & 7 \\
Rango & $1-150$ \\
Reingreso** & $17(2,1 \%)$ \\
Atribuido a cirugía torácica & $15(1,8 \%)$ \\
Atribuido a cirugía extratorácica & $3(0,4 \%)$ \\
\hline
\end{tabular}

*Se registraron casos con dos o más complicaciones. **Un reingreso se atribuyó tanto a una cirugía torácica como extratorácica.

Tabla 8. Mortalidad según lesiones asociadas, tipo de traumatismo y temporalidad de la cirugía por traumatismo torácico

\begin{tabular}{|lccccc|}
\hline & Total & Urgente & Precoz & Diferida & p \\
Total & $54 / 808(6,7 \%)$ & $46 / 474(9,7 \%)$ & $2 / 41(4,9 \%)$ & $6 / 293(2,0 \%)$ & \\
Tipo de TT & & & & \\
TT aislado & $30 / 600(5,0 \%)$ & $26 / 341(7,6 \%)$ & $1 / 32(3,1 \%)$ & $3 / 227(1,3 \%)$ & $0,001^{*}$ \\
$\quad$ Contuso & $4 / 68(5,9 \%)$ & $2 / 20(10,0 \%)$ & $0 / 2(0,0 \%)$ & $2 / 46(4,3 \%)$ & $0,579 *$ \\
Penetrante & $26 / 532(4,9 \%)$ & $24 / 321(7,5 \%)$ & $1 / 30(3,3 \%)$ & $1 / 181(0,6 \%)$ & $<0,001 *$ \\
TT con LA & $24 / 208(11,5 \%)$ & $20 / 133(15,0 \%)$ & $1 / 9(11,1 \%)$ & $3 / 66(4,5 \%)$ & $0,029 *$ \\
Contuso & $14 / 88(15,9 \%)$ & $11 / 48(22,9 \%)$ & $1 / 2(50,0 \%)$ & $2 / 38(5,3 \%)$ & $0,023 *$ \\
Penetrante & $10 / 120(8,3 \%)$ & $9 / 85(10,6 \%)$ & $0 / 7(0,0 \%)$ & $1 / 28(3,6 \%)$ & 0,447 \\
TT con PT & $22 / 164(13,4 \%)$ & $18 / 111(16,2 \%)$ & $1 / 7(14,3 \%)$ & $3 / 46(6,5 \%)$ & 0,104 \\
Contuso & $14 / 69(20,3 \%)$ & $11 / 42(26,2 \%)$ & $1 / 2(50,0 \%)$ & $2 / 25(8,0 \%)$ & 0,109 \\
Penetrante & $8 / 95(8,4 \%)$ & $7 / 69(10,1 \%)$ & $0 / 5(0,0 \%)$ & $1 / 21(4,8 \%)$ & 0,675 \\
\hline
\end{tabular}

*Estadísticamente significativo; TT: Traumatismo torácico; LA: Lesiones asociadas; PT: Subgrupo politraumatizados.

Tabla 9. Mortalidad según tipo de traumatismo torácico y temporalidad de la cirugía por traumatismo torácico

\begin{tabular}{|lccccc|}
\hline & Total & Urgente & Precoz & Diferido & p \\
Total & $54 / 808(6,7 \%)$ & $46 / 474(9,7 \%)$ & $2 / 41(4,9 \%)$ & $6 / 293(2,0 \%)$ & $<0,001^{*}$ \\
Penetrante & $36 / 652(5,5 \%)$ & $33 / 406(8,1 \%)$ & $1 / 37(2,7 \%)$ & $2 / 209(1,0 \%)$ & $<0,001^{*}$ \\
Contuso & $18 / 156(11,5 \%)$ & $13 / 68(19,1 \%)$ & $1 / 4(25,0 \%)$ & $4 / 84(4,8 \%)$ & $0,005 *$ \\
\hline
\end{tabular}

*Estadísticamente significativo. 


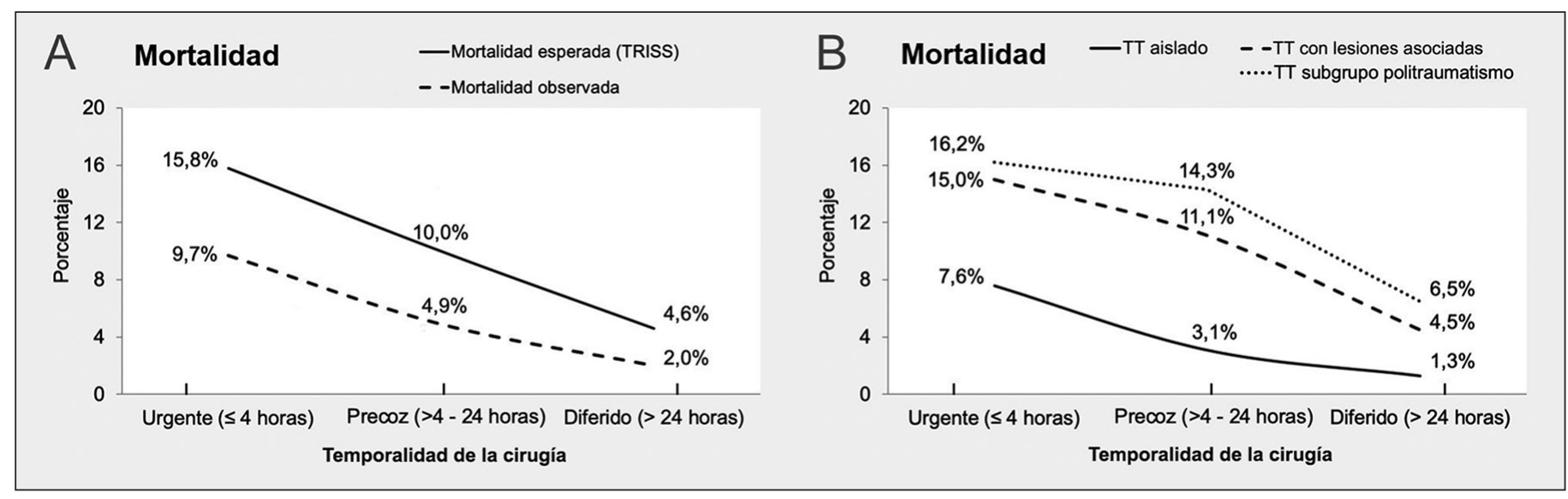

Figura 1. A: Mortalidad esperada (TRISS) y mortalidad observada según la temporalidad de la cirugía por traumatismo torácico. B: Mortalidad según tipo de traumatismo y temporalidad de la cirugía por traumatismo torácico (TT: Traumatismo torácico).

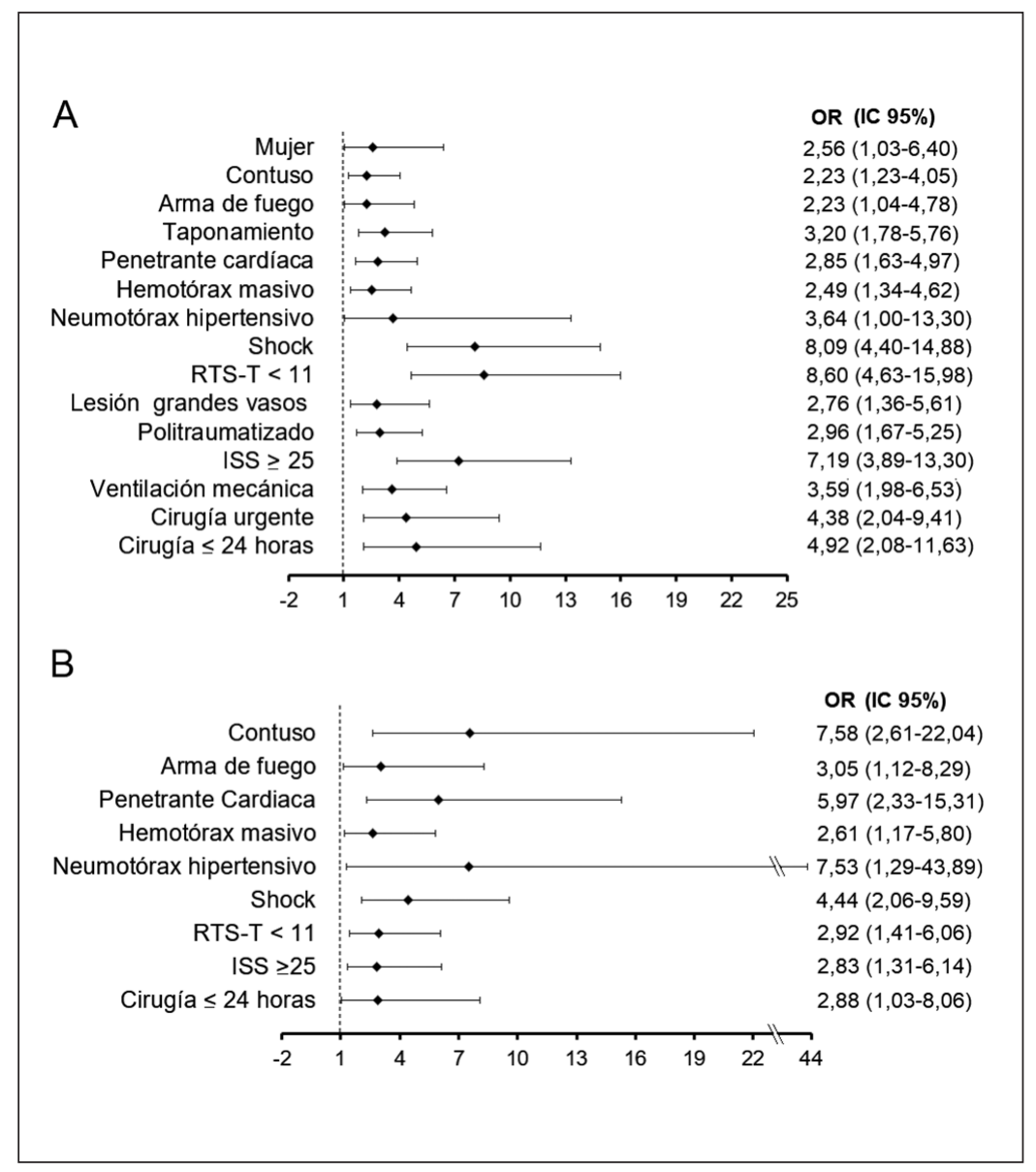

Figura 2. A: Variables estadísticamente significativas asociadas a mortalidad en análisis univariado de cirugías por traumatismo torácico; B: Variables independientemente asociadas a mortalidad en análisis multivariado de cirugías por traumatismo torácico. OR: Odds Ratio; IC: Intervalo de confianza; RTS-T: Revised Trauma Score-Triage; ISS: Injury Severiy Score.
La CTT está indicada generalmente en pacientes con lesiones graves, sin embargo, ciertos casos requieren de un tratamiento quirúrgico independientemente de su gravedad. A pesar de que la mayoría los TT penetrantes pueden ser tratados satisfactoriamente con pleurotomía, en nuestra serie se observó una predominancia de los TT penetrantes operados por sobre los contusos, lo que se condice con series publicadas ${ }^{16-18}$. Cabe destacar que en la serie general de TT de nuestro grupo, el porcentaje de traumatismos penetrantes fue aproximadamente del $60 \%$, proporción que aumenta a un $80 \%$ en los pacientes tratados con $\mathrm{CTT}^{19}$. De forma similar, al comparar el porcentaje de politraumatizados en la serie global de TT se observó un aumento desde un $11,9 \%$ a un $20,3 \%$ en pacientes con $\mathrm{CTT}^{19}$. En relación al mecanismo, este fue principalmente el traumatismo por arma blanca, observándose una baja proporción de traumatismos por armas de fuego en los pacientes con CTT, al compararla con series que reportan una prevalencia de hasta un $40 \%$ de traumatismos por armas de fuego ${ }^{8,16}$, lo que se puede explicar por las características sociodemográficas de nuestra población ${ }^{20}$.

Posterior a un TT, el tratamiento quirúrgico es llevado a cabo en diferentes etapas de la evolución del traumatismo. La cirugía de urgencia ha sido definida como la realizada durante las primeras horas posterior al traumatismo, variando dicho periodo según el autor ${ }^{8,18}$. En nuestro estudio, las CTT urgentes correspondieron aproximadamente al $60 \%$ de las CTT, siendo similar a otras series ${ }^{5,16}$. Las indicaciones de CTT urgente más frecuentemente descritas son el débito alto o progresivo a través de 
la pleurotomía y/o hemotórax masivo, el deterioro del estado hemodinámico, el $\mathrm{TPC}^{4,5,8,16,21}$, entre otros, lo que concuerda con nuestra experiencia. Las lesiones intratorácicas más comúnmente reportadas son las laceraciones pulmonares, lesiones cardíacas, diafragmáticas y de vasos intercostales ${ }^{5,8}$.

En cuanto a la instalación de pleurotomía preoperatoria, al menos un estudio describió pleurotomía en la mayoría de los $\operatorname{casos}^{8}$, similar a lo ocurrido en nuestro análisis. La relevancia de la pleurotomía como tratamiento en el TT puede verse subestimada al analizar exclusivamente el grupo de pacientes con CTT. Al analizar la totalidad de pacientes con pleurotomía en nuestra serie global de TT (4.448 pacientes), del total de pacientes que fueron tratados con pleurotomía sin una lesión torácica que constituyó una indicación de cirugía per se, solo en el 6\% se realizó una CTT por disfunción, complicación o porque la pleurotomía fue insuficiente como tratamiento. De este modo, en el $94 \%$ de estos TT la pleurotomía fue el tratamiento definitivo, lo que concuerda con análisis similares en otros centros, además, este grupo se caracteriza por una mínima morbimortalidad y corta estadía hospitalaria ${ }^{5,22,23}$. En nuestra experiencia los pacientes tratados con pleurotomía tuvieron una mediana de 4 días de estadía hospitalaria.

En relación a la CTT diferida, esta ha sido descrita, entre otras indicaciones, para el tratamiento de hemotórax retenidos, sangrados persistentes, empiemas pleurales, reparaciones de lesiones diafragmáticas y estabilizaciones de pared torácica ${ }^{4,5,13,24}$. En nuestro estudio, las cirugías diferidas constituyeron aproximadamente un tercio de las CTT y fueron motivadas principalmente por complicaciones del hemotórax (retenido, coagulado y/o infectado). Es en las cirugías diferidas donde la VATS (video-assisted thoracoscopic surgery) ha demostrado mayor utilidad, siendo recomendada en grupos seleccionados de pacientes sin deterioro hemodinámico, especialmente con complicaciones del hemotórax y empiema postraumático ${ }^{2,17,25-28}$. Como grupo la reservamos y la recomendamos como abordaje en pacientes sin deterioro hemodinámico, en cirugías diferidas, con estudio imagenológico completo y en los casos en que se han descartado lesiones asociadas potencialmente letales. Además, creemos que el equipo quirúrgico debe ser capacitado y tener experiencia en videotoracoscopía.

En nuestra experiencia en VATS, esta se realizó solo en CTT diferidas, y correspondió aproximadamente al 10\% del total de cirugías, porcentaje similar a otras series ${ }^{4,29}$. En nuestro registro, destacamos dos casos que presentaron sangrado intermitente secundario a una lesión de la arteria mamaria interna por un TT por arma blanca y cumplieron todos los requisitos antes señalados, por lo que fueron operados por este abordaje.

La mortalidad de los pacientes tratados con CTT se ha descrito en un rango entre un $9 \%-11 \%{ }^{5,8}$, considerándose la necesidad de cirugía un predictor significativo de mortalidad por sí mismo ${ }^{30}$. Sin embargo, debido a que constituyen un heterogéneo grupo de lesiones e indicaciones, autores han demostrado una variación de la morbilidad y mortalidad al analizar distintos subgrupos. Onat y cols. $^{8}$ en un análisis univariado de pacientes con TT penetrantes operados de urgencia reportaron un aumento significativo de la mortalidad en lesiones cardíacas y cuando se asociaron a lesiones abdominales. Demirhan y cols. ${ }^{5}$ encontraron un puntaje ISS y una mortalidad significativamente mayor en los pacientes con TT operados de urgencia versus operados no urgentes. Refaely y cols. ${ }^{21}$ en una serie de pacientes ingresados en una unidad de cuidados intensivos con CTT en un periodo menor de $48 \mathrm{~h}$ reportaron una mortalidad del $29 \%$ alcanzando un $45 \%$ en los traumatismos contusos.

En nuestro estudio, debido al alto número de casos, realizamos un análisis de múltiples subgrupos. De estos, se observó una relación directa y coherente en la mortalidad observada según la temporalidad de la cirugía, las lesiones asociadas y el tipo de traumatismo contuso versus penetrante. De esta forma el subgrupo que presentó la mayor mortalidad fueron los pacientes con TT contuso, politraumatizados y con CTT urgente, que alcanzó el 26,2\%, mientras que los casos de TT penetrante, con una lesión aislada y con CTT diferida registraron una mortalidad del $0,6 \%$. La enorme diferencia de la mortalidad demuestra la gran variedad de las lesiones que pueden asociarse al TT y la relevancia de considerar el mayor número posible de características del traumatismo al momento de evaluar los resultados de la cirugía, debido a que, incluso, dentro de un mismo subgrupo de pacientes pueden presentarse pronósticos diferentes.

En concordancia con esto, fueron variables independientemente asociadas a mortalidad la cirugía urgente y precoz, el traumatismo contuso y el politraumatismo grave, los cuales en sumatoria representan al subgrupo de pacientes con la mayor mortalidad observada. Cabe destacar que a pesar de que el traumatismo contuso fue el grupo de menor frecuencia dentro de las CTT, fue la variable con mayor efecto sobre la mortalidad, demostrando la gravedad de dicho grupo de $\mathrm{TT}^{31}$.

Es importante considerar que existen lesiones 
asociadas a TT penetrante que han demostrado tener peor pronóstico, tales como el TPC, el hemotórax masivo y las heridas por armas de fuego, que constituyen subgrupos con características propias y elevada morbilidad y mortalidad ${ }^{32-35}$.

En relación con la participación de los cirujanos de urgencia y cardio y/o torácicos en las CTT urgentes, Doll y cols. ${ }^{36}$, sugieren que la mayoría de las lesiones por TT pueden ser tratadas por cirujanos de urgencia y recomiendan no retrasar la cirugía para ser atendidos por un especialista en pacientes inestables, ya que esto aumentaría el riesgo de mortalidad $^{37}$. En nuestro hospital, todos los pacientes con CTT son seguidos y tratados en el posoperatorio por nuestro equipo, y según nuestro registro aproximadamente en el $85 \%$ de las CTT urgentes hay participación de un cirujano cardio y/o torácico (en el acto quirúrgico, manejo inicial y/o indicaciones), además, la totalidad de las CTT diferidas son realizadas por nuestro equipo. Destacamos que los resultados de nuestro centro se deben en gran medida a la experiencia y entrenamiento de los cirujanos de urgencia, ya que ellos son los responsables del manejo inicial en la gran mayoría de los pacientes con traumatismos. Esto es producto de una cons- tante retroalimentación, evaluación de resultados, capacitación y colaboración entre los cirujanos de urgencia, residentes de cirugía y nuestro equipo en el manejo del TT.

En conclusión, en nuestro medio los pacientes en los que se realizó una CTT se caracterizaron por ser principalmente traumatismos penetrantes y secundarios a lesiones por arma blanca, sin embargo, se observó una elevada heterogeneidad en las indicaciones, hallazgos y lesiones torácicas y extratorácicas en los pacientes tratados con CTT. Múltiples variables fueron relevantes en el análisis de la mortalidad, infiriéndole un carácter de gravedad propio a diferentes grupos de TT.

\section{Responsabilidades éticas}

Protección de personas y animales. Los autores declaran que para esta investigación no se han realizado experimentos en seres humanos ni en animales.

Confidencialidad de los datos. Los autores declaran que en este artículo no aparecen datos de pacientes.

Conflictos de interés: no hay.

\section{Bibliografía}

1. World Health Organization. Injuries and Violence. The Facts 2004. The magnitude and causes of injuries. 2014;20. Disponible en: http://www.who. int/violence_injury_prevention/media/ news/2015/Injury_violence_facts_2014/en [Consultado el 24 de septiembre de 2020].

2. Dennis BM, Bellister SA, Guillamondegui OD. Thoracic Trauma. Surg Clin North Am. 2017;97:1047-64.

3. Veysi VT, Nikolaou VS, Paliobeis C, Efstathopoulos N, Giannoudis PV. Prevalence of chest trauma, associated injuries and mortality: a level I trauma centre experience. Int Orthop. 2009;33:1425-33.

4. Al-Koudmani I, Darwish B, Al-Kateb $\mathrm{K}$, Taifour Y. Chest trauma experience over eleven-year period at al-mouassat university teaching hospital-Damascus: a retrospective review of 888 cases. J Cardiothorac Surg. 2012;19:35. doi: 10.1186/1749-8090-7-35.

5. Demirhan R, Onan B, Oz K, Halazeroglu S. Comprehensive analysis of 4205 patients with chest trauma: a 10-year experience. Interact Cardiovasc Thorac Surg. 2009;9:450-3.

6. Menger R, Telford G, Kim P, Bergey MR, Foreman J, Sarani B, et al. Complications following thoracic trauma managed with tube thoracostomy. Injury 2012;43:46-50.

7. Saaiq M, Shah SA. Thoracic trauma: presentation and management outcome. J Coll Physicians Surg Pak. 2008;18:230-3.

8. Onat S, Ulku R, Avci A, Ates G, Ozcelik C. Urgent thoracotomy for penetrating chest trauma: analysis of 158 patients of a single center. Injury 2011;42:900-4.

9. Baker SP, O'Neill B, Haddon W, Long W. The Injury Severity Score: a method for describing patients with multiple injuries and evaluating emergency care. J Trauma 1974;14:187-96.

10. Champion H, Sacco W, Copes W, Gann D, Gennarelli T, Flanagan M. A revision of the Trauma Score. J Trauma 1989;29:6239.

11. Boyd C, Tolson M, Copes W. Evaluating trauma care: the TRISS method. Trauma Score and the Injury Severity Score. J Trauma 1987;27:370-8.
12. Mica L, Rufibach K, Keel M, Trentz O. The risk of early mortality of polytrauma patients associated to ISS, NISS, APACHE II values and prothrombin time. J Trauma Manag Outcomes 2013;7:6.

13. Meredith JW, Hoth JJ. Thoracic Trauma: When and How to Intervene. Surg Clin North Am. 2007;87:95-118.

14. Rangel-Frausto MS, Pittet D, Costigan M, Hwang T, Davis CS, Wenzel RP. The natural history of the Systemic Inflammatory Response Syndrome (SIRS). A prospective study. JAMA 1995;273:117-23.

15. Carrico CJ, Meakins JL, Marshall JC, Fry D, Maier RV. Multiple-Organ-Failure Syndrome. Arch Surg. 1986;121:196-208.

16. Ahmad T, Ahmed SW, Hussain N, Khan MI. Role of Urgent Thoracotomy in improving the survival of patients with severe chest trauma. J Coll Physicians Surg Pak 2009;19:575-8.

17. Çakmak M, Nail Kandemir M. Study of 433 Operated Cases of Thoracic Trauma. Indian J Surg. 2016;78:477-81.

18. Bertoglio P, Guerrera F, Viti A, Terzi AC, Ruffni E, Lyberis P, et al. Chest drain and 
thoracotomy for chest trauma. J Thorac Dis. 2019;11:186-91.

19. González R, Riquelme A, Fuentes A, Saldías R, Reyes R, Seguel E, et al. Traumatismo torácico: caracterización de hospitalizaciones durante tres décadas. Rev Med Chile 2018;146:196-205.

20. González R, Barra S, Riquelme A, Reyes $\mathrm{R}$, Alarcón F, Seguel E, et al. Traumatismo torácico: análisis de hospitalizaciones según grupo etario. Rev Cir 2021;73: [In press].

21. Refaely Y, Koyfman L, Friger M, Ruderman L, Saleh MA, Sahar G, et al. Clinical Outcome of Urgent Thoracotomy in Patients with Penetrating and Blunt Chest Trauma: A Retrospective Survey. Thorac Cardiovasc Surg. 2018;66:686-92.

22. Kong VY, Sartorius B, Clarke DL. The selective conservative management of penetrating thoracic trauma is still appropriate in the current era. Injury 2015;46:49-53.

23. Menger R, Telford G, Kim P, Bergey MR, Foreman J, Sarani B, et al. Complications following thoracic trauma managed with tube thoracostomy. Injury 2012;43:46-50.

24. Advanced Trauma Life Support. American College of Surgeons. 7th edition. Chicago. 2004. Chapter III. Shock. pp 97-146.

25. Navsaria PH, Vogel RJ, Nicol AJ. Thoracoscopic evacuation of retained posttraumatic hemothorax. Ann Thorac Surg. 2004;78:282-5.

26. De Lesquen H, Avaro JP, Gust L, Ford
RM, Beranger F, Natale C, et al. Surgical management for the first $48 \mathrm{~h}$ following blunt chest trauma: state of the art (excluding vascular injuries). Interact Cardiovasc Thorac Surg. 2015;20:399408.

27. DuBose J, Inaba K, Demetriades D, Scalea TM, O'Connor J, Menaker J, et al. Management of post-traumatic retained hemothorax: a prospective, observational, multicenter AAST study. J Trauma Acute Care Surg. 2012;72:11-22.

28. Wu N, Wu L, Qiu C, Yu Z, Xiang Y, Wang $\mathrm{M}$, et al. A comparison of videoassisted thoracoscopic surgery with open thoracotomy for the management of chest trauma: A systematic review and metaanalysis. World J Surg. 2015;39:940-52.

29. Beshay M, Mertzlufft F, Kottkamp HW, Reymond M, Schmid RA, Branscheid D, et al. Analysis of risk factors in thoracic trauma patients with a comparison of a modern trauma centre: a mono-centre study. World J Emerg Surg. 2020;31;15: 45.

30. Williams M, Carlin AM, Tyburski JG, Blocksom JM, Harvey EH, Steffes CP et al. Predictors of mortality in patients with traumatic diaphragmatic rupture and associated thoracic and/or abdominal injuries. Am Surg. 2004;70:157-62.

31. González R, Riquelme A, Toloza C, Reyes R, Seguel E, Stockins A, Jadue A, et al. Traumatismo torácico contuso. Rev Chil Enferm Respir. 2019;35:96-103.
32. González R, Riquelme A, Fuentes A, Canales J, Seguel E, Stockins A, et al. Traumatismo penetrante cardíaco: caracterización, resultados inmediatos $\mathrm{y}$ variables asociadas a morbilidad y mortalidad en pacientes operados. Rev Cir. 2019;71:245-52.

33. González R, Riquelme A, Toloza C, Reyes R, Seguel E, Stockins A, et al. Hemotórax masivo por traumatismo torácico en pacientes tratados quirúrgicamente. Rev Cir. 2020;72:434-40.

34. González R, Riquelme A, Ávalos M, Reyes R, Seguel E, Stockins A, et al. Traumatismo torácico por arma de fuego. Rev Cir. 2019;72:293-300.

35. González R, Alarcón F, Riquelme A, Reyes R, Barra S, Seguel S. et al. Traumatismo penetrante cardíaco: Evolución de características, gravedad y mortalidad en pacientes tratados quirúrgicos. Rev Cir 2021; 73: [In press].

36. Doll D, Eichler M, Vassiliu P, Boffard K, Pohlemann T, Degiannis E. Penetrating Thoracic Trauma Patients with Gross Physiological Derangement: A Responsibility for the General Surgeon in the Absence of Trauma or Cardiothoracic Surgeon? World J Surg. 2017;41:170-5.

37. Mollberg NM, Tabachnik D, Farjah F, Lin FJ, Vafa A, Abdelhady K, et al. Utilization of cardiothoracic surgeons for operative penetrating thoracic trauma and its impact on clinical outcomes. Ann Thorac Surg. 2013;96:445-50. 